\title{
La producción de conocimiento orientado a la política en Argentina: un análisis de centros de política, organismos internacionales y centros académicos
}

Policy-oriented knowledge production in Argentina: an analysis of policy centers, international organizations

\section{and academic centers}

Mariano Palamidessi, ${ }^{*}$ Jorge Gorostiaga ${ }^{*}$ Claudia Aberbuj

\begin{abstract}
RESUMEN
Este artículo busca caracterizar el trabajo de un grupo de organizaciones que producen conocimiento para influir sobre el diseño de las políticas educativas en la Argentina. El análisis se basa en entrevistas a los responsables de las áreas de educación y en información extraída de las páginas web de tres centros de política, tres organismos internacionales y un centro académico, que se seleccionaron por su fuerte continuidad y presencia en la generación de este tipo de conocimiento desde comienzos de la década de 2000. Se describen y comparan los siete casos de acuerdo con cinco dimensiones: definición de la agenda de producción de conocimiento; financiamiento; conformación de los equipos de trabajo; estrategias de producción de conocimiento; y circulación del conocimiento.
\end{abstract}

Palabras clave: educación, producción de conocimiento, política pública, organizaciones

\section{ABSTRACT}

This article aims at characterizing the work of a group of organizations that produce knowledge in order to influence the design of educational policies in Argentina. The analysis is based on interviews to those responsible for the areas of education and on information extracted from the websites of three policy centers, three international organizations and one academic center, which were selected for their strong continuity and presence in the generation of this type of knowledge since the early 2000s. The seven cases are described and compared according to five dimensions: definition of the knowledge production agenda; financing; conformation of the work teams; knowledge production strategies; and knowledge dissemination.

Key words: education, production of knowledge, public policy, organizations

\section{Introducción}

En este artículo nos proponemos caracterizar el trabajo de un grupo de organizaciones que producen conocimiento para influir

${ }^{*}$ Instituto Nacional de Evaluación Educativa (INEEd), Uruguay; •Universidad Nacional de San Martín, Argentina;" Universidad Nacional de La Plata, Argentina. Contacto: jorgegoros@gmail.com 
sobre el diseño de las políticas educativas en la Argentina. El foco está puesto en tres centros de política, tres organismos internacionales y un centro académico, que se seleccionaron por su fuerte continuidad y presencia en la generación de este tipo de conocimiento desde comienzos de la década de dos mil. El análisis se basa en entrevistas a los responsables de las áreas de educación realizadas entre 2012 y $2013^{1}$ y en información extraída de las páginas web de las instituciones.

En la primera parte, se presenta el marco conceptual del estudio. La segunda sección aborda el desarrollo histórico reciente de este tipo de organizaciones y sus contribuciones a la investigación en educación en la Argentina, con énfasis en las organizaciones elegidas. Una tercera sección describe los casos de las siete agencias seleccionadas de acuerdo con cinco dimensiones de análisis: a) definición de la agenda de producción de conocimiento; b) financiamiento; c) conformación de los equipos de trabajo; d) estrategias de producción de conocimiento; y e) circulación del conocimiento. En la cuarta parte, se comparan las organizaciones de acuerdo con las cinco dimensiones mencionadas.

\section{Las organizaciones productoras de conocimiento orientado a la política}

Por conocimiento orientado a la política (COP) entendemos un tipo de conocimiento elaborado con el fin primordial de influir sobre uno o más aspectos de los procesos de las políticas públicas (Postlethwaite, 1986; Timmer, 1998). Esto incluye distintas instancias de producción, sistematización y difusión de conocimiento para la formulación de las políticas, que abarcan desde posicionamientos orientados a moldear la opinión pública o la visión de un sector particular (como los decisores a nivel gubernamental) hasta la intervención en la implementación de una política pública.

\footnotetext{
${ }^{1}$ Fueron realizadas las siguientes: Gustavo Iaies, CEPP (18/10/12), referida en el texto como Entrevista GI; Florencia Mezzadra, Axel Rivas y Cecilia Veleda, CIPPEC (6/11/12), Entrevista FM, AR y CV; Elena Duro, UNICEF (9/11/12), Entrevista ED; Myriam Southwell, FLACSO (4/12/12), Entrevista MS; Irene Kit, ACEPT (10/12/12), Entrevista IK; Emilio Tenti Fanfani, IIPE-UNESCO (21/11/12), Entrevista ETF; Néstor López, IIPE-UNESCO (6/8/13), Entrevista NL; Darío Pulfer, OEI (30/8/13), Entrevista DP.
} 
Así, el COP se enmarca dentro de la investigación social aplicada o de desarrollo (Richie y Spencer, 1994; OCDE-CERI, 2004), en contraposición a la investigación pura o básica, y responde a una tradición que puede remontarse al menos al siglo XVIII (Wagner et al., 1999). La producción de conocimientos sociales orientada a la política se caracteriza, principalmente, por su interdisciplinariedad, por la defensa o promoción de determinados valores, y por la atención a factores contextuales (Wagner et al., 1999).

Históricamente, la investigación educativa se desarrolló a nivel mundial en torno a dos sistemas institucionales: los organismos centrales de administración y gobierno de los sistemas educativos, y las universidades y las instituciones superiores de formación docente. Mientras que el primero ha tenido una clara función de producir conocimiento útil para la toma de decisiones, el segundo sistema se ha orientado, tradicionalmente, hacia un conocimiento más "puro" o básico. En la segunda mitad del siglo XX emergieron nuevas instituciones vinculadas con la investigación educativa: organizaciones internacionales, organismos de crédito, centros universitarios de investigación aplicada, tanques de pensamiento, centros de investigación y desarrollo vinculados con el mundo empresarial, consultoras, ONG nacionales y de alcance global. Estas nuevas organizaciones producen, mayoritariamente, un conocimiento que busca tener una aplicación o una influencia sobre las políticas y las prácticas educativas, y han sabido articularse -de maneras más o menos institucionalizadascon el Estado y sus demandas y necesidades de conocimiento experto.

Los analistas situados en organizaciones externas al Estado juegan un importante rol en las nuevas formas de gobierno a través de redes, como constructores de alianzas flexibles que atraviesan las fronteras entre lo público y lo privado, la burocracia y el mundo empresarial (Ball y Exley, 2010; Williamson, 2012). En el campo educativo, esto se ha manifestado en el surgimiento de nuevos actores institucionales que intervienen en el proceso de las políticas (como contratistas, asesores, etc.) legitimando determinadas visiones y discursos (Ball, 2008). La articulación de la dimensión nacional con la internacional es otro aspecto clave del trabajo de estas organizaciones, el cual implica muchas veces 
la adaptación de discursos globales de reforma educativa a contextos nacionales. En este sentido, los organismos internacionales y los tanques de pensamiento con ramificaciones mundiales desempeñan un papel cada vez más significativo en un escenario globalizado de producción de políticas educativas (Lessard, 2008).

Dentro de este universo de organizaciones diferenciadas tanto del Estado como de las universidades, ${ }^{2}$ se distinguen tres tipos principales de instituciones que pueden actuar dentro de los contextos nacionales, aunque no necesariamente limitadas a un país determinado:

a) Centros de política, cuya función es la de colaborar con políticas en curso o proponer nuevas políticas en una o más áreas o temas, en algunos casos con vinculaciones directas con partidos políticos.

b) Organismos internacionales, especialmente aquellos con representaciones o sedes locales.

c) Centros académicos no universitarios cuya producción, al menos en parte, responde a las demandas de $-\mathrm{o}$ a la búsqueda de influencia sobre- los procesos de las políticas públicas.

Existe una variedad de funciones vinculadas con la producción, mediación y difusión de conocimiento $-\mathrm{y}$ asignadas típicamente a los tanques de pensamiento- que, en distinta medida, pueden cumplir los tres tipos de organizaciones (Stone, 2000; Thompson, 1994; Uña, 2006):

- ser difusor de ideas nuevas: "traducción” y popularización de ideas y conocimientos nuevos para los que se busca generar consenso (circulación de ideas);

- presentar propuestas de políticas y brindar asesoramiento en

\footnotetext{
${ }^{2}$ Una buena parte de estas organizaciones, incluyendo los centros o fundaciones de política y los centros académicos, han sido asimiladas al concepto de tanque de pensamiento (o think tank, en el original en inglés). Uña (2006) propone una clasificación de tanques de pensamiento, a partir de una definición amplia de estos: "institutos de investigación, organizaciones no gubernamentales y organizaciones privadas... orientados a la investigación en temas públicos o la promoción de políticas públicas con el fin de influir sobre el proceso de formulación de las mismas" (Uña, Cogliandro y Labaqui, 2004: 7) y, basándose en un trabajo de Thompson (1994), los clasifica como: centros de investigación privados, centros académicos, fundaciones políticas y grupos de advocacy. Stone (2000), sin embargo, prefiere reservar el término de think tank para nombrar a los que en nuestra tipología denominamos centros de política.
} 
su implementación, incluyendo la "transferencia de políticas" desde otros contextos nacionales;

- realizar análisis y evaluación de políticas o programas existentes;

- aportar una voz experta ante los medios de comunicación y distintas organizaciones gubernamentales y no gubernamentales;

- generar ámbitos de intercambio y negociación entre dirigentes de distintos ámbitos, llegando a construir en algunos casos "redes de conocimiento";

- ser fuente de reclutamiento de personal para organismos técnicos del Estado.

Siguiendo a Bourdieu (2002), podemos ver a las organizaciones productoras de COP como parte de un campo intelectual, dentro del cual luchan por ejercer influencia con base en la acumulación de un capital específico, en este caso la legitimidad basada en recursos intelectuales (competencias técnicas y conocimiento especializado) para expresar una voz experta sobre las políticas educativas. Su supervivencia en el campo intelectual depende de su forma de gestionar una serie de recursos y de posicionarse frente a las circunstancias propias del contexto en el que operan; en los países en desarrollo suelen enfrentar contextos caracterizados por una serie de elementos limitantes, entre ellos la inestabilidad política y económica, la alta rotación de los responsables de formular políticas y la poca demanda de investigación por parte del Estado (Braun et al., 2006).

Para estas agencias, la definición de la agenda temática ${ }^{3}$ es un paso crucial en el proceso de producción de conocimiento. El impacto sobre las políticas y la posibilidad de sustentabilidad organizacional depende, entre otros factores, de la capacidad de "idear una agenda estratégica de investigación" (Braun et al., 2006: 84), encontrando un balance entre el desarrollo de áreas o "nichos" de especialización y la flexibilidad para responder a las transformaciones del entorno (respuesta a demandas diversas y a cambios en la orientación de las políticas públicas).

\footnotetext{
${ }^{3}$ Esta definición temática implica la selección y problematización de demandas, intereses o problemáticas de algún sector de la sociedad y su constitución como asuntos generales de interés (lo que Oszlak y O’Donnell -1995- denominan “cuestión”). La conversión de asuntos sectoriales en "cuestiones" otorga legitimidad a un tema para ser investigado y debatido, y lo posiciona como problemática con necesidad de solución a través de la política pública.
} 
A su vez, el conocimiento que estas organizaciones producen y ponen en circulación puede tener distintos grados de formalización y asumir distintas formas de externalización (Delvaux y Mangez, 2008): en un polo, se sitúa el conocimiento experiencial, altamente contextual, que tiende a ser oralmente externalizado, ${ }^{4}$ mientras que en el polo opuesto, se encuentra el saber producido de acuerdo con procedimientos científicos y externalizado de forma escrita. Dentro del conocimiento más académicamente formalizado, puede distinguirse entre la producción de estudios propios y la utilización de resultados de investigaciones realizadas por otros (Pons y Van Zanten, 2007), sin excluir combinaciones posibles.

\section{La actividad de las organizaciones productoras de conocimiento orientado a la política en 30 años de democracia}

\section{Organismos internacionales}

Desde la reinstauración de la democracia en 1983 a la actualidad, diversos organismos internacionales han contribuido a la producción de conocimiento sobre educación y han adquirido una presencia significativa -aunque variable- en los debates y en la conformación de la agenda educativa argentina (Galarza, Suasnábar y Merodo, 2007). Entre estas organizaciones, se distinguen dos tipos principales de agencias: las de cooperación, incluyendo organismos del sistema de Naciones Unidas -como el Fondo de las Naciones Unidas para la Infancia (UNICEF) y el Instituto Internacional para el Planeamiento de la Educación (IIPE-UNESCO)-, la Organización de Estados Iberoamericanos (OEI) y la Organización de Estados Americanos (OEA); y las financieras, como el Banco Mundial (BM) y el Banco Interamericano de Desarrollo (BID).

\footnotetext{
${ }^{4}$ Este tipo de conocimiento puede asimilarse al concepto de "saberes de acción", definido como un conocimiento que se deriva de la actuación profesional y es puesto "al servicio de una lógica de acción” (Gather Thurler, 1988, citado en Mosquera, 2006: 141).
} 
UNICEF comenzó a funcionar en la Argentina en 1985. Desde sus inicios desplegó una fuerte actividad de cooperación técnica con el ministerio nacional, los gobiernos provinciales y los organismos no gubernamentales en las diferentes áreas vinculadas al bienestar de la infancia y adolescencia. En ese marco, sus investigaciones sobre la situación y los derechos de los niños y adolescentes, el desarrollo de políticas sociales, las oportunidades educativas en contextos de pobreza y el fracaso escolar lo ubicaron como un espacio constante de referencia. También la actividad de la OEI y de la OEA -que nuclean regionalmente a los Estados y que han tenido, con variaciones a través de los años, un papel importante en la definición de problemas y prioridades de política- expresó un creciente interés por las cuestiones educativas. Sin embargo, en consonancia con su pérdida de gravitación a nivel regional, la presencia e influencia de la OEA en materia educativa en Argentina ha sido decreciente en las últimas tres décadas. Por contraposición, el accionar de la OEI se incrementó por el acompańamiento de los procesos de formulación e implementación de políticas y programas, actividad por la cual promueve y financia proyectos de investigación. La OEI ha mantenido, desde la década de los noventa, una revista especializada en educación (la Revista Iberoamericana de Educación) ${ }^{5}$ y su sede local promovió diversos estudios y publicó diversas series de libros en los que, si bien se privilegia una perspectiva iberoamericana, se abordan problemáticas referidas a la situación de la Argentina (Galarza, Suasnábar y Merodo, 2007).

Los organismos financieros, por su parte, habían visto crecer su influencia hacia fines de los ochenta (Corbalán, 2002). El BM y el BID generaron buena parte del diagnóstico y de las soluciones a la crisis que atravesaban los países latinoamericanos. La agenda de las reformas propuestas incluyó, en un lugar central, a los sistemas educativos. Una parte importante de sus trabajos se centró en diversos aspectos del programa de reformas, tanto en cuestiones programático-prospectivas, como en la revisión y análisis de sus consecuencias (Krawczyk, 2002). En una etapa de crisis fiscal y de debilidad político-institucional de los Estados, estos

\footnotetext{
${ }^{5}$ Sus temáticas se ordenan en función de las agendas de política educativa a nivel regional y las cuestiones sobre las que a la OEI le interesa promover la reflexión y el debate.
} 
organismos alcanzaron una significativa capacidad para movilizar recursos financieros y simbólicos -documentos, diagnósticos, informes y conferencias- que les permitió incidir con fuerza en la conformación de la agenda de la política educativa argentina (Coraggio y Torres, 1999). Sin embargo, la década de dos mil estuvo marcada por un menor protagonismo de estas agencias en la Argentina, llevando a que su actividad se concentrara en la búsqueda de oportunidades para mantener su cartera de crédito. La amplia disponibilidad de recursos durante buena parte de este periodo, el giro ideológico y la concentración de poder borraron del escenario opciones de política educativa que habían sido planteadas por actores como el BM hasta la crisis de 2001 (municipalización, reforma de los estatutos docentes, evaluación de aprendizajes "con consecuencias fuertes", evaluación del desempeño docente, etcétera).

Los cambios de la década de dos mil no impactaron fuertemente sobre la agenda de producción de conocimiento sobre educación por parte de UNICEF. ${ }^{6}$ Pese a que la educación es solo una parte del conjunto de preocupaciones de esta agencia, sus trabajos sistemáticos sobre la situación y la educación de los niños y adolescentes en el país lo han mantenido en el centro de las discusiones de política. Respecto a la OEA, si bien en los primeros años de la década de dos mil, su trabajo estuvo centrado en la promoción de políticas a través de proyectos hemisféricos, cuyas prioridades se establecen a partir de las Cumbres de las Américas y de diagnósticos específicamente construidos a tal fin, su actividad e influencia local continuaron disminuyendo.

En cambio, durante la década de dos mil, la sede local de la OEI ganó un significativo protagonismo. Por un lado, mediante iniciativas regionales como: a) el programa Metas 2012, que fue acompañado por la producción de una serie de materiales que desarrollaron las bases conceptuales del programa, con la inter-

\footnotetext{
${ }^{6}$ Según la responsable del área educativa, UNICEF Argentina se propone estudiar temas que no hayan sido investigados en torno a la temática de la infancia en general y de la educación en particular, con la intención de que "el conocimiento sirva para direccionar políticas en pos de disminuir las brechas de inequidad o de desigualdad". Se intenta "sumar una mínima gota de aporte para llenar un vacío de conocimiento, que apunte a las brechas de desigualdad. No somos una universidad ni somos un centro de generación de conocimiento. Solamente podemos poner tres o cuatro temas anuales que consideramos importantes. Más que eso no podemos en términos de tiempo, recursos, gente, financiamiento" (entrevista ED).
} 
vención de numerosos expertos de España y América Latina; b) la creación de los Institutos de Investigación y Desarrollo (IDIE), que en el caso de la oficina Buenos Aires, siendo la única en la región que tenía un equipo técnico estable, le permitió incorporar nuevos temas de trabajo (infancia, formación docente y alfabetización); y c) por medio de proyectos desarrollados en asociación con otras organizaciones, como en el caso del Sistema de Información Tendencias Educativas de América Latina (SITEAL, gestionado por el IIPE-UNESCO). Por otro, por su rol como administrador de proyectos para el Ministerio de Educación de la Nación, como en el caso del programa Conectar Igualdad. ${ }^{7}$ Esta capacidad de adaptación local a la situación de cada país ha permitido a la OEI sobrellevar la disminución de los fondos y de la influencia política de la cooperación española a partir de la segunda mitad de la década.

Establecida en 1998, la actividad de la Sede Regional Buenos Aires del IIPE-UNESCO durante la década de dos mil consolidó un espacio institucional de referencia en la producción y difusión de conocimiento y en la definición de agendas de debate en la Argentina y en la región. El IIPE aloja el SITEAL (que, como se mencionó antes, se desarrolla en cooperación con la OEI), orientado a la difusión de datos, informes y análisis. ${ }^{8}$ A través de distintos acuerdos, el IIPE realiza programas de asistencia técnica (formación, estudios o consultoría) para satisfacer los requerimientos de gobiernos, organismos e instituciones involucrados en el mejoramiento de la educación.

\section{Centros de políticas}

En el curso de la década de los noventa se constituyó un entramado de fundaciones, consultores, centros de investigación y tanques de pensamiento vinculados crecientemente con organismos internacionales y agencias estatales (Lardone y Donadi, 2006). Con la crisis de las fundaciones vinculadas a partidos políticos

\footnotetext{
${ }^{7}$ Programa que, desde 2010, se propuso -a través de una fuerte inversión del gobierno nacional- la distribución de tres millones de computadoras portátiles a alumnos y docentes de las escuelas de gestión estatal de Educación Secundaria Orientada y de Educación Técnico Profesional, así como de los Institutos de Formación Docente.

${ }^{8}$ www.siteal.iipe-oei.org.
} 
(como la Fundación Plural o la Fundación Andina), que habían alimentado el debate de ideas durante el gobierno de Raúl Alfonsín, nuevos centros de políticas -vinculados con grupos económicos y fundaciones empresarias- asumieron mayor protagonismo como mediadores entre la investigación y la elaboración de políticas (Gorostiaga et al., 2006). Desde mediados de la década de los ochenta, estos centros de políticas habían empezado a ganar espacio a través de la producción de conocimiento y el desarrollo de propuestas de política, posicionándose como voces expertas frente a los medios de comunicación, el Estado y los principales actores de la sociedad civil (Simón y Palamidessi, 2007). Think tanks de inspiración económica liberal -el Instituto de Estudios de la Realidad Argentina y Latinoamericana (IERAL), la Fundación de Investigaciones Económicas Latinoamericanas (FIEL) y, posteriormente, el Grupo Sophia y la Fundación Gobierno y Sociedad- expandieron su actividad e influencia, desarrollando numerosos estudios, diagnósticos y propuestas de política en materia educativa. No obstante, el fuerte protagonismo de miembros de estas agencias en diversas áreas del Estado nacional y provincial -centralmente en el área económica- durante la década de los noventa, su inserción en el ámbito educativo estatal fue marginal, salvo durante la breve gestión de Juan Llach como Ministro de Educación en 2000.

Por otra parte, cabe mencionar la acción de los sindicatos docentes, particularmente la Confederación de Trabajadores de la Educación de la República Argentina y el Sindicato Unificado de Trabajadores de la Educación de la Provincia de Buenos Aires, los cuales, en reacción con lo que consideraban reformas que atacaban a la escuela pública e intentaban debilitar los sindicatos, comenzaron la producción de documentos (algunos basados en investigaciones propias) hacia mediados de los noventa. En una línea similar se ubica el Instituto de Capacitación e Investigación de los Educadores de Córdoba, creado por la Unión de Educadores de esa provincia (UEPC) en 2001, cuya investigación se nuclea, particularmente, alrededor de la temática de "la producción de la (des)igualdad educativa en la provincia de Córdoba".

El giro hacia una retórica estatista de la década del dos mil disminuyó claramente el espacio de influencia de varios centros 
de política. Esto incentivó el cierre de algunas organizaciones -como la Fundación Gobierno y Sociedad- mientras que en otras -IERAL, FIEL- se redujeron o desaparecieron las áreas especializadas en investigación y formulación de propuestas de política educativa. Otras agencias se desestructuraron, en la medida en que sus integrantes se sumaron a la gestión pública, como el Grupo Sophia, en el caso del gobierno de la Ciudad de Buenos Aires. En general, el periodo 2001-2015 no fue fructífero para la investigación en materia educativa de las fundaciones económicas, ya que decayó fuertemente su presencia en el debate y su influencia sobre las políticas. Aun cuando puede haber razones particulares que intervienen en la trayectoria de cada caso, el declive general parece obedecer a la deslegitimación y desplazamiento de la agenda neoliberal. En cambio, otros centros de política creados apenas antes o a partir de la eclosión de la crisis de fin de siglo, lograron abrirse camino. Desde los primeros años de la década de dos mil, el Centro de Implementación de Políticas Públicas para la Equidad y el Crecimiento (CIPPEC) fue construyendo un lugar de relevancia en la arena político-educativa, diversificando los temas y las estrategias de producción de conocimiento y procurando legitimar sus producciones en ámbitos académicos. ${ }^{9}$ Partiendo de un origen asociado a la difusión del movimiento de las charter schools, el área Educación del CIPPEC reformuló rápidamente su agenda a partir de un ambicioso estudio sobre la política educativa en las provincias (Rivas, 2004). Pocos años después, se había posicionado como un centro con voz reconocida, que acompañaba a los organismos del Estado nacional, las provincias y municipios en la formulación e implementación de políticas públicas a través de un importante número de estudios, programas de trabajo e iniciativas, con muy alta presencia en la prensa escrita y con interlocución con actores políticos, sindicales, empresariales, ONG y de medios de comunicación.

Por su parte, la Asociación Civil Educación para Todos (ACEPT) surge a fines de 1999 a partir de la salida de Sergio España

\footnotetext{
${ }^{9}$ El CIPPEC se diseñó a partir de uno de esos modelos representado por el Pioneer Institute de Massachussets, conformado por un grupo de expertos con vocación política: "CIPPEC tenía que ir como por otro camino que era tratar de apoyar al Estado. Había que meterse a trabajar con el Estado en la implementación. No ser tanto un centro de estudios, sino más bien un centro que apoya al Estado en cambios concretos" (entrevista FD, AR y CV).
} 
e Irene Kit de la gestión nacional, donde habían liderado el equipo responsable del Plan Social Educativo. El trabajo que llevó al equipo a volcarse a la producción de conocimiento fue un estudio estadístico sobre repitencia en el primer ciclo de la escuela primaria, investigación que se llevó adelante en 2002 en la provincia de Misiones, por solicitud del Banco Mundial. ACEPT consiguió luego autorización para completar el estudio estadístico con trabajo de campo y ese fue el punto de partida de las dos líneas de acción que ha desarrollado desde entonces: trabajo con información estadística y estudio del currículo real (entrevista IK).

El Centro de Estudios en Políticas Públicas (CEPP), creado en 2003, tiene un origen similar al de ACEPT, ya que se trata de una organización que capitaliza el conocimiento y la expertise adquirida por un equipo tecno-político en su paso por la gestión educativa, en este caso del gobierno de la Alianza. Se trata de un centro cuya misión principal es la generación de conocimiento dirigido a comprender e influir en el ejercicio cotidiano de las políticas públicas. ${ }^{10} \mathrm{El}$ CEPP se fue especializando en armar propuestas de estrategias "a medida", utilizando y haciendo circular el conocimiento y la experiencia disponible a nivel regional. Desde mediados de la década de dos mil, ha desarrollado seminarios de política, estudios y actividades de asesoramiento en diversos países de América Latina (México, Brasil, Ecuador, Chile), así como en algunas provincias argentinas. ${ }^{11}$

\footnotetext{
${ }^{10} \mathrm{El}$ CEPP surge como "una organización en contraste con otras que había. Miraba los problemas desde adentro de los ministerios y no desde afuera. Lo que hacía era pensar por dónde le aprieta el zapato a un tipo que está sentado en un despacho y que tiene que tomar decisiones. Se trata de ver cómo ayudás a un funcionario a pensar lo que él no ve y a reconstituir una lógica más estructural de cómo mirar su sistema educativo y lo que está haciendo" (entrevista GI).

${ }^{11}$ Otro ejemplo del desarrollo de centros de política es el del Laboratorio de Políticas Públicas (LPP), fundado también en 2003, pero cuya agenda de investigación en educación se fue diluyendo con el paso del tiempo. En el marco del Foro Latinoamericano de Políticas Educativas, una iniciativa regional interinstitucional de la cual forma parte el LPP, en el periodo 2007-2014 se produjeron cuatro documentos sobre Argentina enfocados en la participación de la sociedad civil en el gobierno de la educación, las políticas de formación docente, el derecho a la educación y la educación intercultural bilingüe. El LPP se define como un "centro de estudios orientado a la investigación para la incidencia en políticas públicas" y comprometido con "la democratización del Estado y los espacios públicos, así como [con] la construcción de prácticas políticas, sociales y culturales inclusivas y con un sentido transformador" (http://lppargentina.org.ar/sample-page/, julio 2014).
} 


\section{Centros académicos}

Desde comienzos de la década de los ochenta, entre los centros académicos productores de COP se destacó el Área de Educación de la Facultad Latinoamericana de Ciencias Sociales (FLACSO), sede Argentina. Creada en 1982, bajo la dirección de Juan Carlos Tedesco y liderada, posteriormente, por Cecilia Braslavsky, el área desempeñó un rol clave en el desarrollo de la investigación educativa por medio de estudios que -en diálogo con las ciencias sociales- renovaron los temas y preocupaciones del debate durante las décadas de los ochenta y los noventa. ${ }^{12}$ Algunos de los trabajos producidos en la FLACSO tuvieron una fuerte y amplia influencia en el campo intelectual de la educación, como los que abordaron la revisión crítica de la educación durante la dictadura, la discriminación educativa en la Argentina o las problemáticas de la administración y la calidad del sistema educativo. Durante el proceso de reformas en la década de los noventa, varios de sus investigadores pasaron a la gestión estatal. Otros centros académicos, como el Centro de Estudios de Estado y Sociedad (CEDES) y el Centro de Estudios de Población (CENEP), también generaron una serie de insumos relevantes para el debate y la formulación de políticas educativas durante la década de los noventa, aunque con un impacto significativamente menor que FLACSO.

No obstante, en la década de dos mil, la influencia de la FLACSO en los debates de política disminuyó. Diversos factores parecen haber influido en esta pérdida de posición relativa en el campo. Si bien durante esa década llegaron al cargo de Ministros de Educación intelectuales como Daniel Filmus o Juan Carlos Tedesco -que marcaron los orígenes del Área de Educación- y diversos miembros de la institución colaboraron con las gestiones ministeriales, la agenda de producción de la institución tendió a perder unidad y dirección. La producción más sistemática del área se concentró en estudios sobre la educación media, la formación docente y el análisis de los efectos de las reformas educativas de la década de los noventa, asumiendo un renovado interés por el análisis de las desigualdades sociales y escolares (Tiramonti,

\footnotetext{
${ }^{12}$ Esta lógica articulaba buena parte de la producción de tesis de maestría, las actividades de consultoría y la realización de proyectos de investigación con financiamiento externo.
} 
2004). Sin poder eludir el clima de polarización creciente que caracterizó a la política argentina a partir de la segunda mitad de la década de dos mil, sus investigadores se dividieron entre un acompañamiento conceptual-ideológico al proyecto educativo oficial y una crítica pública de sus orientaciones. Organizaciones como el CENEP y el CEDES, por su parte, concentraron su rol en el de asiento institucional de investigadores y becarios del sistema nacional de ciencia y tecnología, colaborando, en algunos casos, en las investigaciones de UNICEF.

\section{Las lógicas de producción de conocimiento orientado a la política y las características organizacionales}

En esta sección se describen y analizan las lógicas de producción de conocimientos en cada una de las siete organizaciones escogidas durante el periodo 2001-2013, considerando el modo en que: a) definen su agenda de producción de conocimiento; b) financian las actividades de producción de conocimiento; c) conforman sus equipos de trabajo; d) desarrollan estrategias de producción de conocimiento $y, e)$ hacen circular sus productos. ${ }^{13}$

\section{UNICEF sede Argentina}

La agenda de producción de conocimiento de UNICEF se orienta al desarrollo de estudios para informar y direccionar políticas que buscan disminuir las brechas de inequidad o de desigualdad en materia de derechos de la infancia y la adolescencia en general y de la educación en particular. UNICEF define planes de cuatro años de duración a partir de lineamientos regionales y temáticas prioritarias. A nivel nacional, los programas de cooperación son presentados, por sector, a cada autoridad ministerial y a su equi-

\footnotetext{
${ }^{13}$ En este apartado se analizará la unidad que en cada organización se dedica a la producción de COP en educación. En los casos del CEPP y de EpT se analiza la organización en su conjunto; en el caso de CIPPEC, el Programa de Educación, que es parte del Área de Desarrollo Social; dentro de UNICEF Argentina, el programa de Inclusión y Calidad Educativa para Niños, Niñas y Adolescentes; en el IIPE Buenos Aires, la actividad de la Unidad de Investigación y del equipo del SITEAL; en OEI Argentina, el Programa de Educación; y en la FLACSO Argentina, el Área de Educación.
} 
po, quienes deben aprobarlos. Cuando el programa está aprobado, UNICEF identifica los vacíos de conocimiento e información existentes y decide, para cada tema, qué organización o investigador podría llevar adelante el estudio. Los proyectos duran, en promedio, un año y medio, y el proceso total insume alrededor de dos años. El diseño de investigación se realiza conjuntamente entre el equipo experto convocado y la coordinación del área de educación de UNICEF. Los estudios se financian a través de los fondos aportados por individuos que cooperan con la organización.

UNICEF Argentina no cuenta con un equipo estable dedicado a actividades de investigación, sino con un coordinador general, quien contrata equipos pertenecientes a universidades, centros de investigación o fundaciones para desarrollar los distintos proyectos. Usualmente, la búsqueda y selección de equipos o investigadores se realiza a través de consultas informales a especialistas de confianza o profesionales reconocidos. En la selección se privilegia las características del investigador que lidera el equipo ${ }^{14}$ por sobre las de la institución a la que pertenece.

La producción de conocimiento que realiza UNICEF responde, principalmente, a una lógica de investigación empírica y tiene un alcance nacional. Una vez que el equipo finaliza el estudio, entrega los resultados obtenidos a la coordinación y se revisan en conjunto. En esa instancia se define si se debe profundizar en algún punto o agregar algún componente. El proceso termina en un informe, acompañado de propuestas de políticas, en formato impreso o digital. A partir de allí, suele desplegarse un proceso de cooperación y diálogo entre UNICEF y los ministerios con miras a instalar o modificar políticas públicas.

\section{OEl Argentina}

La sede de la OEI en Buenos Aires desarrolla su agenda de producción de información y de conocimiento en materia educativa como parte de sus actividades de cooperación, acompańamiento de la gestión y consejo experto a nivel nacional y provincial. Esta agenda

\footnotetext{
${ }^{14}$ Los requisitos son que tengan "perfiles muy altos como investigadores, que sepan manejar técnicas cuantitativas y cualitativas, que conozcan en profundidad el tema y que tengan mucha experiencia en manejo de equipos de trabajo" (entrevista ED).
} 
se va conformando a partir de prioridades fijadas a nivel regional, pero buscando también responder a las necesidades y demandas locales. A partir de mediados de la década de dos mil, los temas de la agenda de la OEI tendieron a encuadrarse en el plan Metas 2021:

Desde infancia, formación docente en evaluación en valores, alfabetización de adultos, todos los temas TIC... Si apuramos un poco y decimos cuáles son las más importantes: infancia y universalización de secundaria. Ahí están nuestras apuestas, nuestros focos, que son las que también vamos planteando al gobierno... Donde más inversión y más esfuerzo hay que hacer para garantizar por lo menos el perfil de democratización, que es lo que está planteado en las Metas (entrevista DP).

Uno de sus principales dispositivos de producción, que desarrolla junto al IIPE-UNESCO, es el SITEAL, que realiza informes anuales que responden a prioridades y demandas de la agenda de la OEI, en temas como infancia, adolescencia, educación de pueblos originarios y afro-descendientes, y nuevas tecnologías. Para algunas temáticas, la oficina Buenos Aires asume también la coordinación de la Revista Iberoamericana de Educación.

Además de las cuotas obligatorias y aportes voluntarios que efectúan los gobiernos de los Estados Miembros, para sustentar las actividades de sistematización de información e investigación la OEI busca recursos mediante la presentación de proyectos a concursos, lo que le ha permitido obtener financiamiento de la Unión Europea y otras fuentes. Pero una parte importante de la financiación de la OEI y de sus actividades de producción de conocimientos se cubre mediante la administración de programas y proyectos para los gobiernos. Dentro de los proyectos en los cuales ha cooperado con el Ministerio de Educación de la Nación en los últimos años -asumiendo funciones de administración e implementación - se destaca el programa Conectar Igualdad, que se articuló con la creación de IBERTIC. ${ }^{15}$

${ }^{15}$ El IBERTIC es una iniciativa de la OEI de carácter regional en el marco de las Metas 2021. Su objetivo es "brindar asistencia técnica especializada a los países iberoamericanos ... para el desarrollo de acciones de diagnóstico, identificación, orientación, prospectiva, formación, evaluación y otras líneas de intervención que colaboren en la aplicación eficaz de las TIC" (sitio web ibertic.org). IBERTIC, con sede en Buenos Aires, se desarrolla en tres áreas específicas: Investigación, Formación y Evaluación. 
La oficina de Buenos Aires de la OEI cuenta con un equipo estable de 12 integrantes. El equipo está encargado de realizar asistencia técnica, supervisar o dirigir una investigación, aunque es común que para diversos proyectos se contrate a expertos o equipos externos. La producción de COP que se realiza tiene un alcance nacional, ya que sus objetivos remiten a la cooperación con el Estado tanto nacional como provinciales. ${ }^{16}$ No obstante, su alcance se proyecta a nivel regional al establecer líneas de acción con los gobiernos, como en el caso de METAS 2021.

\section{IIPE-UNESCO Buenos Aires}

Durante la década de dos mil, las actividades de producción de conocimiento del IIPE se organizaron en función de distintos tipos de agendas, con lógicas de financiamiento y de trabajo diferenciadas. Una parte de tal actividad se vinculó con la atención de demandas de organizaciones que recurrieron al IIPE por la confianza técnica-intelectual en sus equipos y la legitimidad que brinda la UNESCO. ${ }^{17}$ Pero también se desarrollaron proyectos que partieron de los intereses académicos de los integrantes del área, como un estudio sobre docentes, que se sostuvo a lo largo de varios años y se replicó en varios países de la región con el apoyo de diversos ministerios. Finalmente, hay proyectos de producción de información y conocimiento que han logrado mantenerse en el tiempo y seguir una agenda definida por la organización con un financiamiento de más largo plazo. En síntesis, la agenda de producción de conocimiento del IIPE se estructuró en la negociación entre la identidad institucional, ${ }^{18}$ los intereses y prioridades -intelectuales y de política- de sus investigadores y las necesidades de las organizaciones demandantes o de sus financiadores.

\footnotetext{
16 "Nuestra responsabilidad es lo nacional; entonces, ya cuando cumplimos medianamente demanda y objetivo, la idea era avanzar con provincias en el armado de planes de mediano a largo plazo a partir de datos de información leída un poco más localizadamente" (entrevista DP).

17 "Hay proyectos totalmente generados afuera, que son resultado de una demanda que llega a la institución... El IIPE fue ganando un prestigio, un nombre que hace que sea demandado, que sea una firma... Lo que se busca muchas veces es cierta legitimidad para los ministerios. La UNESCO es un nombre... Y si aparte tienen buena calidad... Pero les interesa el sello UNESCO" (entrevista ETF).

18 "Creo que esta oficina tuvo una identidad clara. Hay un posicionamiento institucional frente al debate regional... la reivindicación de lo público, la idea de justicia, la educación como derecho" (entrevista NL).
} 
El IIPE ha llevado a cabo proyectos de investigación con distintos horizontes de financiamiento. Hubo proyectos de dos o tres ańos (como los desarrollados con el auspicio de la Fundación Ford), otros de pocos meses y otros que han logrado generar una base de recursos sostenible a lo largo de varios años (como el SITEAL, compartido con la OEI). En varios casos, un proyecto comenzó financiado por una institución interesada y luego fue sumando el aporte de otras organizaciones. En los proyectos basados en demandas externas, la principal estrategia de financiamiento ha sido a través de los actores demandantes de los proyectos, ya sean empresas, fundaciones o gobiernos.

Durante la década de dos mil, se estructuraron dos equipos dedicados a la producción de conocimiento, cada uno con una forma de organización específica. Por un lado, la Unidad de Investigación giró en torno a un coordinador, quien contrataba investigadores para la realización de cada proyecto; ${ }^{19}$ por el otro, el SITEAL se basó en un equipo que desplegó cierta continuidad a lo largo de los años, atravesando distintos proyectos y líneas de trabajo y que llegó a contar con 10-12 integrantes estables.

La producción de conocimiento sigue una lógica de investigación empírica cualitativa y cuantitativa. En el caso de los estudios a pedido de países u organizaciones, el coordinador negocia el diseño del proyecto de investigación y las temáticas de interés. Aunque el proceso finaliza con la producción de un informe con los resultados y propuestas de políticas, en algunos casos se realiza una instancia de presentación de resultados. En el caso de los programas de producción más continuos -como el SITEAL-, los productos finales son libros, informes o documentos impresos o disponibles en la página web de la institución.

Sus investigaciones, en general, tienen un alcance nacional, pero en ocasiones algunos países de la región solicitan a los equipos que realicen estudios o repliquen investigaciones realizadas en Argentina en sus propios territorios. No obstante, la intención no es la circulación regional del conocimiento, sino la respuesta

\footnotetext{
${ }^{19}$ La dificultad de conformar un equipo estable de investigación aparece como un elemento que complica el armado de una agenda menos regida por las demandas externas: "Si no hacés una inversión de base, tenés poca capacidad de poner condiciones o de incluir temáticas en la agenda” (entrevista ETF).
} 
a pedidos locales de diversos países de la región. Su labor finaliza al entregar el informe de investigación, por lo que no existen instancias posteriores de seguimiento o monitoreo del uso de la información producida.

\section{CIPPEC}

La agenda de producción de conocimiento del Programa Educación del CIPPEC se constituye con base en pedidos o demandas externas y a los temas de interés definidos por su equipo, y se materializa en proyectos de trabajo que se ordenan en función de una planificación trienal y una anual. ${ }^{20} \mathrm{La}$ trienal define líneas estratégicas y la anual sirve para considerar las dinámicas y oportunidades que presenta la coyuntura y concretar las líneas generales. ${ }^{21}$ Esa planificación debe dejar también lugar para atender demandas no previstas, principalmente de parte del Estado, que se considera que pueden tener un alto impacto en la orientación de las políticas educativas. Sin embargo, la organización ha construido capacidades que le permiten realizar con continuidad proyectos de cierta envergadura vinculados a sus intereses. ${ }^{22}$

Las estrategias de financiamiento de sus investigaciones son diversas. Para aquellos proyectos que responden a intereses del Programa se buscan recursos a través de la presentación a concursos de fundaciones internacionales, o de organismos de cooperación internacionales, multilaterales o nacionales (como en el caso

\footnotetext{
20 "El CIPPEC es una institución fuerte, que tiene una marca propia, que tiene áreas institucionales fuertes, un área de comunicación fuerte, y que intenta distinguirse de la consultora, es decir, no ser una institución que trabaja a demanda... Cada programa tiene una lógica interna, tiene objetivos, tiene un plan de trabajo, se propone hacer proyectos para cambiar o apoyar ciertos cambios e intentamos buscar recursos para hacer eso. Partir de una agenda y mantener al mínimo posible el rol de consultoría. A veces es necesario porque trae recursos económicos, pero no como lógica de intervención. En esta última etapa se solidificó bastante como una institución que trata de cuidar con quién se mete para no perder prestigio" (entrevista FD, AR y CV).

${ }^{21}$ Existe un proceso institucionalizado de aprobación de proyectos. Ningún proyecto puede ser presentado a un donante sin ser aprobado internamente por el Comité Ejecutivo (integrado por seis o siete directores que analizan cada proyecto) en una defensa por parte del programa en términos de lineamientos estratégicos. De este modo, se busca construir coherencia a nivel institucional.

22 "Hace bastante que tenemos nuestra agenda y salimos a buscar financiamiento para eso. No es a la inversa. Sucede a la inversa en algunos casos en que quizás alguna provincia nos viene a solicitar algún asesoramiento técnico en algún tema y lo evaluamos en función de nuestra expertise y de nuestras posibilidades... Cuando las empresas nos vienen a buscar para algo, con alguna demanda particular, intentamos reformular esa demanda" (entrevista FD, AR y CV).
} 
de la Embajada de Finlandia). Para los proyectos que responden a demandas externas, el financiamiento proviene del actor demandante del proyecto (gobierno nacional, gobiernos provinciales, empresas) o de una institución que funciona como financiadora (por ejemplo, el BID).

El Programa posee un equipo estable que se mantiene entre cinco y diez personas. ${ }^{23}$ Se contratan expertos externos -en general, especialistas que hayan tenido algún vínculo con CIPPEC-, en caso de que el equipo permanente no pueda atender las demandas o de requerir una expertise muy específica. El proceso de selección para la incorporación de miembros al equipo es uno de los más formalizados, sobre todo para los puestos junior. Además de entrevistas, se suele tomar algún ejercicio para evaluar competencias específicas. En el caso de los cargos de senior, se prioriza el conocimiento directo y prolongado de la persona.

La lógica de la producción de conocimientos del Programa Educación varía según el tipo de proyecto. Los proyectos de apoyo a la gestión estatal tienden a responder a una lógica de traducción de acuerdos académicos y transferencia política. Usualmente, se construyen consensos con actores del ámbito académico y se ponen a disposición de los funcionarios para que puedan hacer uso de esos conocimientos. Asimismo, se sistematiza y codifica conocimiento en estado práctico de los agentes estatales. Los proyectos de investigación sobre una temática específica -que, por lo general, son solicitados por el Estado para comenzar a pensar políticas públicas en una determinada línea- siguen también una lógica de transferencia política: el CIPPEC indaga qué se está planteando y desarrollando en otros países en referencia a ese tema y sistematiza ese conocimiento en un informe orientado a informar la toma de decisiones. Por último, están los proyectos de estudio y monitoreo de políticas públicas, en los que prima la investigación empírica, tanto en la generación de datos como en la sistematización de información ya existente.

El producto final de sus proyectos suele ser un informe, un libro (impreso o digital) o un texto audiovisual; el tipo de producto se vincula con los propósitos de los proyectos y los desti-

\footnotetext{
${ }^{23}$ En 2012, estaba conformado por seis integrantes y el núcleo del equipo no tenía una formación de grado en educación, sino en otras ciencias sociales.
} 
natarios de los resultados. En el caso en que los destinatarios de los resultados sean actores gubernamentales, se realizan reuniones para discutir el conocimiento producido y las propuestas de política. En otros casos, se realizan presentaciones o se publica el material en la página web del CIPPEC.

La agenda del Programa Educación se orienta, fundamentalmente, hacia problemas y actores nacionales. A pesar del objetivo explícito de trabajar junto al Estado en la implementación de políticas y no ser solo un centro de estudios, el Programa no realiza un seguimiento formal del uso que hacen los destinatarios de los conocimientos producidos o de la implementación de las propuestas de políticas. Si hay algún tipo de seguimiento de la incidencia del trabajo es, principalmente, a través de los medios de comunicación o del diálogo informal con diversos actores.

\section{CEPP}

El primer programa que implementó el CEPP fue el Diálogo Regional de Políticas Públicas, que - con apoyo del BID- reunía regularmente a todos los viceministros de educación de América Latina para poner en común problemáticas existentes en cada país y discutir las posibles estrategias de resolución. Este programa acompañó el surgimiento de la organización. En esa primera etapa, la agenda de producción de conocimiento del CEPP giró en torno a la identificación y la conceptualización de los temas que iban emergiendo en las reuniones de viceministros. Al finalizar cada una de las reuniones se realizaban informes, en los que se definían los temas y ejes estratégicos a partir de los problemas identificados en el intercambio colectivo. Estas actas eran el insumo para identificar tendencias y problemas comunes, y proponer marcos conceptuales y estrategias de resolución que circulaban en los diversos países de la región.

De este modo, el CEPP se fue convirtiendo en una institución que dialogaba en forma continua con autoridades educativas de la región y que fue armando un "catálogo" de problemas educativos que enfrentaban los tomadores de decisión y sus posibles soluciones. La construcción de esta red de información sobre las políticas "en acción" les permitió convertirse en una fuente de consulta a nivel regional. La expertise central del CEPP se formó en el diálogo 
con los gobiernos y con la producción de conocimiento vinculados con la resolución de problemas de gestión. No obstante, hacia el final de la década de dos mil, su agenda de producción de conocimientos tendió a balancear más el trabajo por demanda con la elección de algunos temas por parte de la organización. ${ }^{24}$

El CEPP se financia, principalmente, a partir de los aportes del actor demandante del proyecto, o a través de proyectos donde una tercera institución asume el rol de financiador. Ejemplos del primer caso son el Programa Regional de Políticas Públicas, solicitado y financiado por el Banco Interamericano de Desarrollo (BID), las evaluaciones de sistemas educativos provinciales o los proyectos de asistencia técnica y evaluación de diversas escuelas en Argentina. El segundo caso suele darse en los proyectos solicitados por gobiernos provinciales o nacionales -que son usualmente financiados por organismos de cooperación internacional o fundaciones- $y$ en los proyectos que surgen por iniciativa propia.

En cuanto al equipo de trabajo, se compone de $10-12$ profesionales estables. La coordinación de los proyectos está a cargo de un integrante del equipo. Los profesionales externos que se contratan suelen ser especialistas en la temática del proyecto o en alguna metodología o técnica de investigación. Para su contratación, se privilegia la experiencia en la gestión pública o una aproximación profesional, más que académica. El CEPP es una de las pocas organizaciones analizadas -junto a CIPPEC- donde se realiza un proceso de selección de personal formalizado. Para ello, se realizan entrevistas y los postulantes son evaluados para medir su desempeño en tareas específicas.

El CEPP construye COP en diálogo con expertos y con actores gubernamentales, empresas, fundaciones y sindicatos de diversos países de América Latina, respondiendo principalmente a una lógica de transferencia de políticas. Al definirse una temática o problemática sobre la que se trabajará, se designa a un miembro

\footnotetext{
24 "Al principio surgían mucho más de las demandas; aparecía un laburo en equis temas y nosotros nos íbamos metiendo, le encontrábamos la punta. Hoy hay un combo distinto: a nosotros nos interesa meternos en tal tema y buscamos inversores o gobiernos que quieran entrarle a tal tema. Empezamos a pensar más dónde nos parece que hay un tema estratégico para meterse. El CEPP ha ganado bastante prestigio en ese mundo. Cada vez más podemos hacer los proyectos que queremos y no para lo que nos buscan" (entrevista GI).
} 
del equipo para que coordine el proyecto. El coordinador debe interiorizarse del tema, contactarse con algunos expertos o consultores y construir un estado del arte a partir de la sistematización y análisis de estrategias seguidas por distintos países frente al mismo problema y mediante la revisión de bibliografía especializada. El equipo analiza el material producido y diseńa lineamientos o propuestas. El producto resultante del trabajo es un informe o documento de trabajo que contiene propuestas de políticas. El último paso del proceso suele ser reunirse con los gobiernos o instituciones para ofrecerles -o entregarles, en el caso de ser productos demandados- las propuestas de política. En el estilo de trabajo del CEPP, estas propuestas son filtradas, centralmente, por criterios de viabilidad de la implementación. Sobre aquellos temas que el CEPP tiene especial interés se realiza un seguimiento más o menos sistemático del proceso. Para los temas que no son centrales, el seguimiento -cuando se realiza- es informal y se desarrolla mediante el diálogo continuo con los gobiernos y actores relevantes.

\section{ACEPT}

La agenda de producción de ACEPT se vincula con la expertise desarrollada por sus miembros fundadores en la experiencia del Plan Social Educativo durante la década de los noventa. ${ }^{25} \mathrm{~A}$ partir de esa experiencia, el equipo construyó una metodología de trabajo que vincula el análisis cuantitativo de información estadística y el estudio del currículum real. ${ }^{26} \mathrm{Al}$ tratarse de un equipo con una expertise, la agenda de trabajo se centra en ella y se ordena en función de las demandas específicas de diversos gobiernos para la producción de diagnósticos, recomendaciones o intervenciones sobre esos temas.

ACEPT ha desarrollado proyectos para gobiernos nacionales de América Central y para diversas provincias argentinas. El finan-

\footnotetext{
${ }^{25}$ EpT surge asociada a una idea central que identifica y cohesiona a la organización: "que es posible superar el fracaso escolar" (entrevista IK).

26 "Una línea tiene que ver con usar la información estadística para identificar los cuellos de botella en términos macro, pero además las unidades educativas que explican la mayor cantidad de ese cuello de botella. Los promedios encubren disparidades. El tipo de comportamiento de esas variables tiene mucha variancia entre escuelas y algunas veces dentro de la misma escuela. La producción de conocimiento tenía que ver con usar la herramienta estadística para grandes mapas y pequeños mapas, tipo Google. Y la otra, es la línea de trabajo con el currículum real..." (entrevista IK).
} 
ciamiento proviene del actor demandante del proyecto -mayormente, gobiernos sub-nacionales-. En otros casos, los proyectos involucran una institución financiadora; esta situación suele darse en proyectos solicitados o dirigidos a países que no cuentan con los fondos para financiarlos y donde se busca la cooperación de organismos internacionales como UNICEF, OEI o UNESCO.

ACEPT posee un núcleo de ocho o nueve profesionales estables -sociólogos, pedagogos y especialistas en sistemas- que contrata equipos de trabajo específicos para realizar los proyectos; los miembros de este núcleo son los responsables de coordinar los proyectos. Los sociólogos se dedican, principalmente, a las tareas relacionadas con la sistematización y análisis de información estadística, y los pedagogos a tareas de análisis de currículum real y la formulación de recomendaciones. El número de profesionales contratados ad-hoc alcanza, regularmente, a 15-20.

El tipo predominante de producción de conocimiento se asocia con la investigación empírica. Los análisis de estadística descriptiva que realizan se basan en los datos censales o de gestión de los ministerios. Para el análisis del currículum real, el equipo construye instrumentos de recolección de información que se procesa mediante diversas estrategias de análisis. El conocimiento producido se presenta en la forma de archivos digitales, documentos e informes. Mucho de este conocimiento se encuentra publicado en Internet, mediante documentos o informes en páginas webs institucionales o a través de sistemas de consulta en línea con gráficos dinámicos.

La producción de ACEPT tiene alcance regional, ya que su trabajo se orienta a desarrollar el mismo tipo de conocimiento con distintos gobiernos nacionales de la región y poner en vínculo ese conocimiento producido. Con los resultados de las investigaciones se hacen devoluciones presenciales a los gobiernos -en algunos casos, de países latinoamericanos, en otros casos, gobiernos provinciales- y también a las escuelas, cuando hubo participación de ellas. Una vez terminados los proyectos, el equipo se propone "transferir la metodología, o sea, ubicar dentro de cada gobierno quién se puede hacer cargo y pasarlo" (entrevista IK). 


\section{FLACSO Argentina}

A partir de 2000, se produjo en FLACSO un importante crecimiento en la oferta de formación del Área Educación (vinculada, en parte, al auge de los cursos virtuales) y, con ello, un aumento de recursos y del número de proyectos y docentes-investigadores. ${ }^{27}$ Esta dinámica de crecimiento afectó la capacidad de estructurar una agenda de producción con sentido estratégico. ${ }^{28} \mathrm{De}$ este modo, durante los últimos 15 años, la producción de conocimiento tendió a diversificarse, crecientemente, según los temas y las especialidades de los investigadores principales que componen el Área y a alinearse a las actividades de formación presencial y virtual. Puede verse como una organización que, de configurarse casi como un centro de política en la década de los noventa, ha ido virando a un centro académico que adopta una forma de funcionamiento asimilable a la de un departamento universitario.

Pese a que el núcleo principal de la actividad de investigación del Área Educación gira en torno a los principales proyectos de los investigadores, también incorpora proyectos que responden a demandas externas vinculados, sobre todo, con la asistencia técnica. En lo que respecta a la producción de COP, este ha tendido a mantenerse estable desde principios de la década de dos mil, pero ocupando un lugar algo marginal respecto a la producción académica. ${ }^{29}$ Estas investigaciones, incluyendo evaluaciones de programas y políticas, se realizan en el marco de convenios de

27 “En los primeros años de 2000 el área pasó de tener tres investigadores a tener $16 \ldots$ En un punto también nos fuimos nutriendo de los vaivenes institucionales de otros lugares, como las entradas y salidas de colegas y de equipos formados del Ministerio de Educación Nacional, del Ministerio de Educación de la Ciudad de Buenos Aires y demás. Resultamos beneficiados por la gente que necesitó otros lugares nuevos donde recalar" (entrevista MS).

28 "Hasta 2000, veníamos de un área todavía muy estructurada en torno a pocos investigadores y que actuaba como colectivo. En general, nos llegaban demandas de asistencia técnica y consultoría y el Área llevaba adelante eso más o menos en términos de grupo. Pero en el ciclo que se inicia después de 2000 empieza a haber muchos más investigadores ... Entonces ya no es tanto la producción del Área estrictamente o los diálogos que el Área tiene con el Ministerio de Educación Nacional o con los ministerios provinciales, sino que se empezó a realizar en torno a nombres o a figuras fuertes" (entrevista MS).

${ }^{29}$ La lógica de producción de conocimiento del Área Educación responde, centralmente, al modelo académico de investigación empírica. También la difusión de resultados de investigación tiende a seguir las pautas de la lógica académica y se materializa en productos de diverso formato como informes, artículos académicos y de divulgación, libros y materiales de docencia. 
asistencia técnica con el ministerio de educación nacional, ministerios provinciales y otras instituciones como el sindicato docente de Córdoba (UEPC), son financiadas por las instituciones demandantes y no suelen tener una circulación abierta ni mecanismos de validación establecidos. Al mismo tiempo, el diálogo con los actores políticos de la educación parece haber decaído desde 2009-2010, sin que exista un posicionamiento unificado del Área frente a las políticas en curso. Se utilizan diversas estrategias para financiar las actividades de investigación. Los proyectos ligados a los temas de especialidad de los investigadores principales tienen dos importantes fuentes de financiación: la presentación de los proyectos a concurso (de agencias nacionales e internacionales) y los recursos obtenidos a través de las actividades de formación (mayormente, cursos virtuales). En el caso de los proyectos que responden a demandas externas, la financiación proviene, en general, de la institución demandante.

El Área tiene un equipo de investigadores principales estable, que define y contrata un equipo específico para cada estudio. Los equipos que se conforman en torno a cada investigador principal suelen integrarse con profesionales o investigadores noveles que ya formaban parte de algún proyecto de la institución en materia de docencia, con investigadores noveles convocados ad-hoc o graduados de sus posgrados. Cada investigador principal y su equipo trabajan en actividades de docencia e investigación y, muchas veces, combinando más de un asiento institucional.

Todos los investigadores principales tienen credenciales académicas de posgrado y poseen experiencia en tareas de docencia e investigación. La selección de los investigadores incorporados responde a un proceso informal y descentralizado. Un cambio significativo de los últimos años ha sido la incorporación de becarios -tanto del sistema nacional de ciencia y tecnología como del de la Provincia de Buenos Aires- los que realizan tareas de investigación, docencia y asistencia técnica. 


\section{Análisis comparado de las lógicas de producción de conocimiento orientado a la política}

\section{Definición de agenda}

Es posible construir un continuum en el que se distribuyen estas organizaciones en función de la estrategia de definición de agenda de producción de $\mathrm{COP} .{ }^{30}$ En uno de los extremos se ubicarían las que definen su agenda en función a su misión y propósitos institucionales y una planificación que se desarrolla en diálogo con la agenda nacional de política educativa; tal el caso de UNICEF. En el otro extremo se ubicarían las organizaciones que definen su agenda fundamentalmente en función a demandas externas, donde podrían ubicarse buena parte de los trabajos del IIPE y FLACSO. En distintos puntos intermedios se encuentran los Centros de Política, la OEI y la producción del IIPE vinculada al Proyecto SITEAL, donde la construcción de agenda es el resultado, en mayor o menor medida, de una negociación entre su misión y propósitos y las demandas externas. CEPP y CIPPEC son las únicas organizaciones que señalan un proceso de "internalización" de la agenda, en el cual han ido pasando de meramente responder a demandas externas, a un modelo de mayor autonomía en la construcción de sus prioridades y orientaciones de producción de COP.

\section{Estrategias de financiamiento}

Existen, en los casos analizados, diversas estrategias de financiamiento de los proyectos de generación de COP. Es posible modelizar tres tipos principales: financiamiento atado a un proyecto específico, financiamiento para el trabajo del área/centro en general, financiamiento a través de otras actividades realizadas por la organización (por ejemplo, enseñanza o asistencia técnica). ${ }^{31}$

\footnotetext{
${ }^{30}$ Cabe aclarar que ninguna institución define su agenda exclusivamente a partir de demandas externas o considerando únicamente su misión y objetivos; en todos los casos, se trata de énfasis y tendencias, siempre existen combinaciones y negociaciones de significado y definición.

${ }^{31}$ Cabe aclarar, que ninguna de las organizaciones, salvo UNICEF, utiliza una sola estrategia de financiación, sino que combina con diversos énfasis las distintas estrategias; no obstante, en cada caso predomina una de ellas.
} 
La estrategia de financiamiento atado a un proyecto específico es predominante en la producción de COP de los Centros de Política estudiados (CIPPEC, CEPP y ACEPT), así como del IIPE y parte de los proyectos de la FLACSO y de la OEI. En estos casos, el financiamiento proviene tanto de la propia institución demandante del conocimiento, como de una institución que se incorpore al proceso específicamente como financiadora (un ejemplo de este caso son las presentaciones a concursos de proyectos específicos). El caso de UNICEF, y en buena medida de la OEI, poseen un financiamiento para el trabajo del área en general que no está ligado a proyectos específicos, ya que cuentan con un presupuesto predefinido en función del cual establecen -en consulta con el gobierno nacional o provinciales- los proyectos a implementar. Por último, el financiamiento a través de otras actividades que realiza la institución, como de enseñanza o asistencia técnica, forma parte de las estrategias utilizadas en FLACSO.

\section{Equipos de trabajo}

Existen dos modelos principales de conformación de equipos de trabajo en las organizaciones analizadas. Por un lado, hay áreas de educación que están compuestas por un solo coordinador que convoca y contrata equipos de investigación ad hoc para cada proyecto. Suelen ser equipos ya conformados y liderados por algún investigador cercano a la organización o formar parte de alguna institución experta en la temática a abordar, a través de quienes se realiza la contratación. Responden a este modelo tanto UNICEF como el equipo de la Unidad de Investigación del IIPE.

Por otro lado, hay organizaciones que poseen equipos estables, más o menos numerosos, que concentran las principales tareas del proceso de producción de conocimiento o su coordinación. Para cubrir todos los proyectos y el propio proceso de producción de COP, en muchos casos deben complementar sus equipos estables con especialistas contratados ad hoc; la relación entre equipo estable y contrataciones adicionales varía de acuerdo con el caso. Responden a este modelo la OEI, los tres centros de política, el equipo del IIPE vinculado a SITEAL y la FLACSO.

El modelo de conformación de equipo responde a diversas variables, entre ellas, a la diversidad de temáticas que la organización 
aborda en materia de producción de conocimiento, al propio financiamiento de las organizaciones y a la posibilidad que poseen para definir el uso del presupuesto con el que cuentan. Todas las organizaciones deben asegurar un financiamiento continuo que garantice el sostén de la estructura estable, lo que significa que parte de la búsqueda de financiamiento y, en consecuencia, de los proyectos que se incluyen en la agenda de trabajo se vinculan con la necesidad de reproducción de la propia estructura.

\section{Lógicas de producción de conocimiento}

En los casos analizados, pueden identificare tres lógicas de producción de conocimiento orientado a la política: transferencia de políticas, investigación empírica y traducción de acuerdos académicos. En algunas de las organizaciones predomina fuertemente alguna de las lógicas, mientras que en otras se alterna entre ellas. La lógica de transferencia de políticas se impone fuertemente en el trabajo del CEPP y forma parte de las estrategias utilizadas por el CIPPEC. Por su parte, la lógica de investigación empírica es predominante en el trabajo de ACEPT, de FLACSO y de los tres organismos internacionales analizados (UNICEF, OEI e IIPE) y forma parte también de las estrategias utilizadas por el CIPPEC. La traducción de acuerdos académicos forma parte de las estrategias utilizadas por el CIPPEC y el CEPP. A su vez, en algunas de ellas (CEPP, CIPPEC, $\mathrm{OEI})$ tiene un peso importante la lógica de generación de conocimiento experiencial, externalizado muchas veces en forma oral.

\section{Circulación de conocimiento}

En cuanto a los productos en los que materializa el conocimiento producido, predomina el informe o documento de trabajo unido, en algunas ocasiones, a propuestas de política. También se generan productos diferenciales que se vinculan a la naturaleza del conocimiento producido y a los destinatarios que intentan alcanzar. Ejemplos destacados son: los libros y audiovisuales de CIPPEC y FLACSO; los sistemas de consulta en línea de indicadores, con gráficos dinámicos, de EpT; los libros y bases de datos producidos por el IIPE, y las revistas editadas por OEI. 
Los principales destinatarios del trabajo son los Ministerios de Educación, ya sea de nivel nacional como jurisdiccional. La mayor diferencia radica en la composición del conjunto de Ministerios de Educación con los que cada institución trabaja. El CEPP y ACEPT poseen un alcance regional, ya que asisten a Ministerios de Educación de diversos países de Latinoamérica; si bien no trabajan con el Ministerio Nacional argentino, sí lo hacen con algunos ministerios provinciales. El CIPPEC y FLACSO, por el contrario, tienen como principal interlocutor al Ministerio $\mathrm{Na}$ cional argentino y trabajan también con algunos ministerios provinciales, pero no asisten a ministerios de otros países. UNICEF y OEI trabajan con el Ministerio de Educación Nacional y con varios ministerios provinciales. El IIPE trabaja con distintos $\mathrm{Mi}-$ nisterios de Educación de la región y con el Ministerio Nacional argentino; OEI y el IIPE, a su vez, producen información, a través del SITEAL, para uso de los diversos ministerios jurisdiccionales y nacionales. En segunda instancia las organizaciones tienen como demandantes de COP a fundaciones, ONG, empresas y diversas instituciones del sistema educativo argentino.

\section{Conclusiones}

Entre las agencias productoras de COP en educación que operaron en la primera década de 2000 , se han analizado las áreas educativas de siete de ellas: tres organismos internacionales (UNICEF, IIPE y OEI), tres centros políticos (CEPP, CIPPEC y ACEPT) y un centro académico (FLACSO). Como mostramos, las agendas temáticas tienden a surgir de una negociación entre los objetivos propios del área o su misión con las demandas de otras instituciones, lo que se vincula con un financiamiento que está predominantemente atado a proyectos específicos y que es de origen externo. Los equipos de trabajo suelen ser estables, pero pequeños. En términos de las lógicas de producción de conocimiento, predomina la producción empírica de tipo académico, pero la transferencia de políticas, la traducción de acuerdos académicos y la generación de conocimiento experiencial tienen un lugar significativo. Los interlocutores principales son los ministerios de educación, sean provinciales o nacionales (de Argentina o de otros países de la región) y los resultados de los procesos de generación de COP suelen expresarse en informes con recomendaciones de política. 
Así como sucedió con el nuevo escenario sociopolítico de la década de los noventa, las tendencias y procesos que se dieron en la primera década de dos mil en Argentina reconfiguraron el mapa de las agencias productoras de COP con una redefinición de sus roles y grados de influencia en la política educativa. El cambio de clima político e ideológico que comenzó a configurarse en los primeros años de la década de dos mil se conjugó con importantes transformaciones en los estilos de gobierno del área educativa, con un Estado Nacional con agenda política propia que se materializó en la promulgación de diversas leyes educativas y en la implementación de diversos programas de llegada nacional.

En ese nuevo contexto, hubo una importante tendencia hacia la reconversión o reposicionamiento de muchas de las organizaciones existentes, principalmente de los centros académicos y los organismos internacionales con sede en Argentina, con excepción de UNICEF, cuya agenda no parece haber sido afectada por el cambio de década quizás debido a la independencia financiera de la que goza. $\mathrm{El}$ posicionamiento de IIPE-UNESCO y OEI, por su parte, pareció basarse en la capacidad para generar proyectos con los gobiernos a partir de ciertos nichos de expertise (por ejemplo, en temas como TIC o en la generación de bases de datos). En los tres casos, parece decisiva la legitimidad de las organizaciones madre (UNESCO, UNICEF y OEI) como interlocutores de los funcionarios y frente a otras organizaciones financiadoras como fundaciones internacionales.

En segundo lugar, varios centros de política y fundaciones económicas existentes, constructoras de agendas de política relevantes durante la década de los noventa, tendieron a desaparecer o a reducir sus áreas educativas. En su lugar, surgieron nuevos centros de política, fundados en muchos de los casos por funcionarios salidos de la gestión, que ampliaron su campo de influencia y circulación de conocimientos a mercados regionales. Los casos de CIPPEC, CEPP y ACEPT ilustran cómo desde 2000 los centros de política tienden a adoptar un rol de acompañamiento técnico al Estado, antes que el de promotores de una determinada visión política que direccione las medidas educativas.

Por último, el protagonismo de FLACSO como productor de COP durante la década de los noventa se diluyó durante los últimos años. Esto parece vincularse con una agenda de trabajo y posicionamiento político menos unificado a partir de la incorporación de más investigadores; y una lógica de funcionamiento 
más afín a la de otros centros académicos o incluso similar a la de un departamento universitario.

En síntesis, el relativo protagonismo y el tipo de actividad de agencias no estatales durante la primera década del siglo XXI se vinculó con un conjunto de factores: la fuerza de la agenda de política educativa del gobierno nacional; las entradas y salidas de grupos de analistas de los ministerios nacional y provinciales; el nuevo clima ideológico imperante, y la persistente debilidad de estructuras estatales para la generación de COP.

\section{Referencias bibliográficas}

Ball, S. J. y Exley, S. "Making policy with 'good ideas': Policy networks and the 'intellectuals' of New Labour", en Journal of education policy, 25 (2), 2010, pp. 151-169.

Ball, S. J. "New philanthropy, new networks and new governance in education", en Political studies, 56 (4), 2008, pp. 747-765.

Bourdieu, P. Campo de poder, campo intelectual. Itinerario de un concepto, Buenos Aires, Montressor, 2002.

Braun, M., Mariana, C., Ducoté, N. y Weyrauch, V. 'Lejos de Thinktanklandia: los institutos de investigación de políticas en los países en desarrollo", en A. Garcé y G. Uńa (comps.), Think tanks y politicas públicas en Latinoamérica: dinámicas globales y realidades regionales, Buenos Aires, Prometeo Libros, 2006.

Cogliandro, G., Labaqui, J. y Uña, G. Politicas públicas y toma de decisiones: Los think tanks en Argentina, Buenos Aires, Fundación Konrad Adenauer, 2004.

Coraggio, J. L. y Torres, R. M. La educación según el Banco Mundial: un análisis de sus propuestas y métodos, Buenos Aires, Centro de Estudios Multidisciplinarios, 1999.

Corbalán, A. El Banco Mundial: intervención y disciplinamiento, Buenos Aires, Editorial Biblos, 2002.

Delvauz, B. y Mangez, E. "Towards a sociology of the knowledge-policy relation", en Literature Review, Integrative Report, Proyect Knowledge and Policy, Unión Europea, 2008, Fecha de acceso 8 de noviembre de 2014. Disponible en http://knowandpol.eu/IMG/pdf/literature_sythesis_final_version_english.pdf. 
Galarza, D., Susnábar, C. y Merodo, A. "Los organismos internacionales e intergubernamentales", en Palamidessi, M., Suasnábar, C. y Galarza, D. (comps.), Educación, conocimiento y politica. Argentina, 1983-2003, Buenos Aires, Manantial, 2007.

Gorostiaga, J., Pini M., Donini A. y Ginsburg, M. “The steering of educational research policy in neoliberal times: The case of Argentina”, en J. Ozga, T. Seddon y T. Popkewitz (eds.), World Yearbook of Education. Education Research and Policy, London, Routledge Falmer, 2006.

Krawczyk, N. "La reforma educativa en América Latina desde la perspectiva de los organismos multilaterales", en Revista Mexicana de Investigación Educativa, 7 (16), 2002, pp. 627-663.

Lardone, M. y Donadi, L. "Construyendo consenso. La modernización del Estado y los mecanismos de articulación entre Bancos Multilaterales de Desarrollo y Think Tanks en Argentina”, en A. Garcé y G. Uña (comps.), Think tanks y politicas públicas en Latinoamérica: dinámicas globales y realidades regionales, Buenos Aires, Prometeo Libros, 2006.

Lessard, C. "Recherche et politiques educatives", en A. Van Zanten (ed.), Dictionnaire de l'éducation, Paris, PUF, 2008, pp. 560-564.

Mosquera, C. "Conocimiento científico y saberes de acción en el Trabajo Social: sobrevaloraciones, desconocimientos y revaloraciones", ponencia presentada en el seminario permanente de docentes del Trabajo Social, Fundación Universitaria Monserrate Programa del Trabajo Social, Bogotá, 2006.

OCDE-CERI. Revisión Nacional de Investigación y Desarrollo Educativo, Reporte de los Examinadores sobre México, 2004. Disponible en https://www.oecd.org/edu/ceri/32496490. pdf. Fecha de acceso 4 de marzo de 2015.

Oszlak, O. y O’donnell, G. "Estado y políticas estatales en América Latina: hacia una estrategia de investigación”, en $R e$ des, 2 (4), 1995, pp. 99-128.

Pons, X., y van Zanten, A. "Knowledge circulation, regulation and governance", en Proyect Knowledge and Policy, Unión Europea, 2007. Disponible en http://www.knowandpol.eu/ IMG/pdf/lr.tr.pons_vanzanten.eng.pdf. Fecha de acceso 14 de mayo de 2014. 
Postlethwaite, T. N. "Policy-oriented research in education", en Oxford Review of Education 12 (2), 1986, pp. 135-151.

Richie, J. y Spencer. L. (1994): Qualitative Data Analysis for Applied Policy Research, en Bryman, A. y Burgess, R. G. (eds.), Analyzing Qualitative Data, Londres, Taylor y Francis, 1994.

Rivas, A. Gobernar la educación. Estudio comparado sobre el poder y la educación en las provincias argentinas, Buenos Aires, Granica, 2004.

Simón, J. y Palamidessi, M. "Las fundaciones financiadas por el empresariado", en Palamidessi, M., Suasnábar, C. y Galarza, D. (Comps.), Educación, conocimiento y politica. Argentina, 1983-2003, Buenos Aires, FLACSO-Manantial, 2007.

Stone, D. "Non-governmental policy transfer: the strategies of independent policy institutes", en Governance, 13 (1), 2000, pp. 45-70.

Thompson, A. Think tanks en la Argentina. Conocimiento, instituciones y politica, Buenos Aires, CEDES, 1994. Fecha de acceso, 5 de agosto de 2012. Disponible en <http://168.96.200.17/ ar/libros/argentina/cedes/thom1.rtf>.

Timmer, C. P. "Adding value through policy-oriented research: reflections of a scholar-practitioner", en Impact Assessment Discussion paper No. 4, Washington DC, International Food Policy Research Institute, 1998.

Tiramonti, G. (Ed.). La trama de la desigualdad educativa: mutaciones recientes en la escuela media, Buenos Aires, Ediciones Manantial, 2004.

Uña, G. "Think tanks en Argentina: sobreviviendo a la tensión entre la participación y la permanencia”, en A. Garcé y G. Uña (comps.), Think tanks y politicas públicas en Latinoamérica: dinámicas globales y realidades regionales, Buenos Aires, Prometeo Libros, 2006.

Wagner, P., Hirschon Weiss, C., Wittrock, B. y Wollman, H. Ciencias sociales y Estados modernos: experiencias nacionales e incidencias teóricas, México, Fondo de Cultura Económica, 1999.

Williamson, B. "Centrifugal schooling: Third sector policy networks and the reassembling of curriculum policy in England”, en Journal of Education Policy, 27 (6), 2012, pp. $775-794$. 